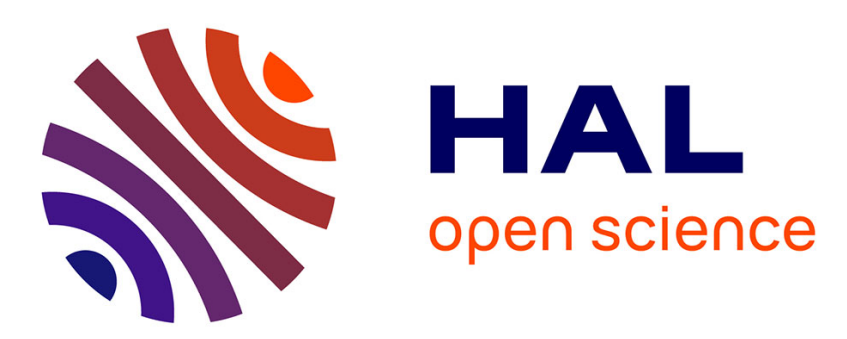

\title{
How Memorizing Positions or Directions Affects Gesture Learning?
}

Bruno Fruchard, Eric Lecolinet, Olivier Chapuis

\section{To cite this version:}

Bruno Fruchard, Eric Lecolinet, Olivier Chapuis. How Memorizing Positions or Directions Affects Gesture Learning?. Proceedings of the 2018 International Conference on Interactive Surfaces and Spaces, Nov 2018, Tokyo, Japan. pp.107-114, 10.1145/3279778.3279787 . hal-01891436v2

\section{HAL Id: hal-01891436 \\ https://hal.science/hal-01891436v2}

Submitted on 3 Dec 2018

HAL is a multi-disciplinary open access archive for the deposit and dissemination of scientific research documents, whether they are published or not. The documents may come from teaching and research institutions in France or abroad, or from public or private research centers.
L'archive ouverte pluridisciplinaire HAL, est destinée au dépôt et à la diffusion de documents scientifiques de niveau recherche, publiés ou non, émanant des établissements d'enseignement et de recherche français ou étrangers, des laboratoires publics ou privés. 


\section{How Memorizing Positions or Directions Affects Gesture Learning?}

\author{
Bruno Fruchard ${ }^{1,2}$ \\ ${ }^{1}$ LTCI, Télécom ParisTech, \\ Université Paris-Saclay \\ F-75013 Paris, France
}

\section{Olivier Chapuis ${ }^{2}$}

\begin{abstract}
Various techniques have been proposed to faster command selection. Many of them either rely on directional gestures (e.g. Marking menus) or pointing gestures using a spatiallystable arrangement of items (e.g. FastTap). Both types of techniques are known to leverage memorization, but not necessarily for the same reasons. In this paper, we investigate whether using directions or positions affects gesture learning. Our study shows that, while recall rates are not significantly different, participants used the novice mode more often and spent more time while learning commands with directional gestures, and they also reported more physical and mental efforts. Moreover, this study also highlights the importance of semantic relationships between gestural commands and reports on the memorization strategies that were elaborated by the participants.
\end{abstract}

\section{CCS Concepts}

- Human-centered computing $\rightarrow$ Gestural input;

\section{Author Keywords}

Memorization; spatial memory; pointing; gestures; command selection; user study.

\section{INTRODUCTION}

To select commands, users typically navigate user interfaces and recognize the name (or the icon) of the command they want to trigger. Using gestural shortcuts, they can also rely on recall. As they already know the action to perform, and do not need to search for the desired command and move the pointer to its location, they can generally go faster. Gestural shortcuts can rely on positions when spatially-stable arrangements of items are used [18, 28], or directions [20,36], and are assumed to favor memorization $[13,25]$. One might wonder, however, how these two approaches affect gesture learning and whether one performs better than the other.

Learning spatial positions is by definition based on spatial memory, the effectiveness of which has been demonstrated

Bruno Fruchard, Eric Lecolinet \& Olivier Chapuis. How Memorizing Positions or Directions Affects Gesture Learning?, In ISS '18: Proceedings of the 2018 International Conference on Interactive Surfaces and Spaces, 107-114, ACM, November 2018.

(C) ACM, 2018. This is the author's version of the work. It is posted here by permission of ACM for your personal use. Not for redistribution. The definitive version is published in ISS '18, November 25-28, 2018, Tokyo, Japan. doi: $10.1145 / 3279778.3279787$

\author{
${ }^{2}$ LRI, Univ. Paris-Sud, CNRS, \\ Inria, Université Paris-Saclay \\ F-91405 Orsay, France
}

in many studies $[8,16,21]$. The placement of commands (and their relative positions) is therefore particularly important [11]. However, it is unclear today whether learning directions relies so much on spatial memory, or whether other types of memorization come into play. Moreover, in both cases, the movements induced by selection actions are likely to exploit muscular memory (or procedural memory) [30] as well as semantic memory [31]. Gestures can indeed be associated with concepts (for example, plus/minus or good/bad or up/down) or everyday actions (turn a page to the left vs. to the right).

Marking menus [20] introduced two ways of performing gestures, either in novice or expert mode. The novice mode provides the users with a visual guide that shows the gestures to be performed $[5,20]$, which helps them to learn by rehearsing these gestures. Once the users can recall them, they can be performed in expert mode, i.e., without a visual guide. Yet, it is unclear how much time users spend to learn with the novice mode and the strategies they use to do so. As a recent study showed, participants are able to elaborate strategies to memorize efficiently without being instructed [13].

In this paper ${ }^{1}$, using a novice to expert procedure that consists in letting users rehearse command selections to memorize them $[17,20]$, we compare the effect of using positions vs. directions on command memorization and study what strategies users elaborate. We present a user study that took place over three sessions over a period of one to two weeks. Participants had to memorize a set of 16 commands (out of a total of 32 possible commands), placed hierarchically in menus containing 8 elements, for each of the two modalities (positions or directions).

The results indicate high memorization performance in both cases with $81.7 \%$ (directions) vs. $84.2 \%$ (positions) of correct selections after the 2 nd session ( 24 hours after the 1 st session), and $53.6 \%$ (directions) vs. $57.1 \%$ (positions) after the 3rd session (one to two weeks after the 1 st session). We did not find significant differences between conditions. However, our analysis reveals slight advantages of using positions about the learning curve and the effort induced to memorize. The subjective opinions of users, gathered in a questionnaire, also point to the same direction. Finally, our study shows that participants adopted similar strategies in both conditions.

\footnotetext{
${ }^{1}$ This paper is an improved english version of a paper [14] that has been accepted at the French HCI conference IHM '18.
} 
We first present the related work and focus on the learning of commands. We then describe the interaction techniques we used and our user study. Finally, we discuss the results obtained and suggest improvements that could be made to graphical interfaces to encourage the use of mnemonic methods by users.

\section{RELATED WORK}

Gestural interaction has been extensively studied in the literature $[4,6,9,20]$. However, most studies focus on performance, and there is comparatively much less work on the memorization of gestures [1, 22, 27]. A possible explanation is that memorization studies are challenging to perform (time-consuming and difficult to design). Nevertheless, they are important as users can not efficiently trigger shortcuts (at least in expert mode) if they have difficulties learning them. In this section, we focus on the memorization of positional and directional gestures.

Position. Using pointing (i.e. positional gestures), users typically select command by clicking on buttons or menu or list items. Interestingly, they can learn the positions of items by using their spatial memory which is assumed to improve long-term memorization [2, 25] and fasten command selection. Consistent spatial interfaces [16, 28, 29] offer a way to promote it and thus help users find commands. By offering the possibility for users to place commands depending on their preferences, it also helps them recall efficiently in the longterm [25]. Such interfaces can provide a novice mode in which the names (or icons) of the items are displayed and an expert mode for users that can recall their positions without being guided visually [18].

Spatial landmarks can also improve the learning of spatial positions [33], especially if their number is large [32]. Natural spatial landmarks on the skin of a user (e.g., birthmark) are, for instance, useful when placing and recalling commands directly on the forearm [7]. Spatial cues of a 3D environment can also be used to facilitate the memorization of commands, e.g., in VR systems or the context of smart home interaction as in [24]. This last study showed that participants obtain high recall rates for a relatively large number of commands (48 commands) and that they were able to remember most of them for at least two months.

Direction. Once the user has learned a set of gestures, he can perform them quickly without being guided. Several studies showed that such gestures could be efficiently memorized (e.g., $[3,13,26])$ and that they provide advantages over keyboard shortcuts [1]. User customization can also improve memorization [22], as well as the degree of effort needed for performing gestures [10]. However, inducing efforts may also make the technique unpleasant.

Marking menus [20] use compound directional marks to navigate hierarchies of commands. This technique builds on two notable advantages: it is based on simple directional movements and provides the same gestures in novice and expert mode to leverage implicit learning. The interaction techniques used in our study draws heavily on Simple-mark menus [37], a

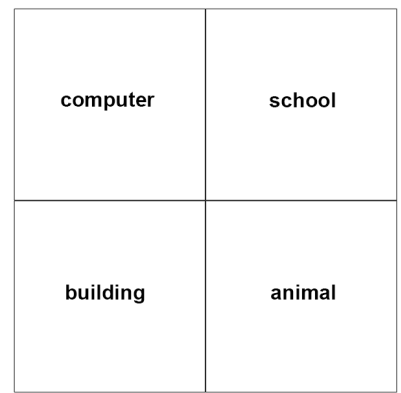

(a)

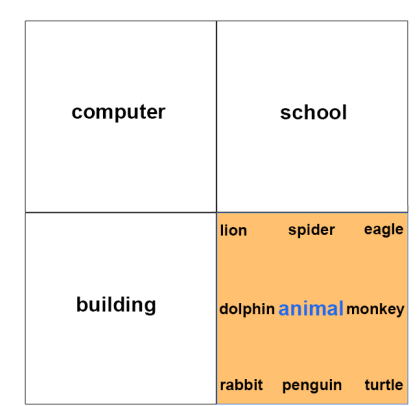

(b)
Figure 1. Representation of menus and commands in the context of selections by pointing in novice mode (the text is magnified on the illustrations to be readable). Each of the four menus is represented by an interaction zone (a). When a menu is selected (here the "animal" menu), the eight commands it contains are displayed (b).

variant of the Marking menus that is less error-prone by using successive selections to navigate a hierarchy of menus.

Several techniques in the literature proposed to combine both positions and directions modalities to define gestural interactions $[12,36,38]$. They proved to be efficient to trigger many commands, and users seemed able to memorize them efficiently [38]. However, few studies have considered comparing them so far [13, 24]. This last study [24] showed superior performance for positional gestures but in a specific context (3D environment using the method of loci [35] ), which combined various factors and did not evaluate the respective effect of each of them. In this paper, we compare positional and directional gestures more formally to investigate whether this difference affects the learning of gestures.

\section{INTERACTION TECHNIQUES}

The interaction techniques for performing positional and directional gestures were developed on a tablet to force direct interaction because this makes it possible to differentiate more clearly the movements involved in both cases (movements may be more similar using a mouse). We now describe both techniques.

Position. Usually, users select commands by clicking buttons or items. To leverage spatial memory, we use an interaction technique inspired by $[18,28]$ that associates each command with a unique position in space. A menu is represented by a rectangular area (Figure 1-a) in which the commands are contained. The commands are placed next to spatial cues to help memorization [32] (i.e., the corners and edges of the menu, Figure 1-b). To select a command, the user first selects a menu by clicking within its interaction area, then on the desired item.

Users must enter the novice mode to see the labels of the commands. They perform this action by pressing the menu area for one second. Such a selection can be canceled by clicking outside menus. Once the novice mode is activated, the names of the (four) menus are constantly displayed in the center of their interaction zones (Figure 1-a). A menu is highlighted in orange once selected, and its commands are displayed along its edges (Figure 1-b). Commands are activated by clicking on them. 


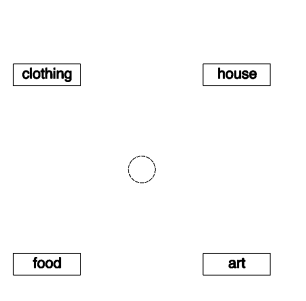

(a)

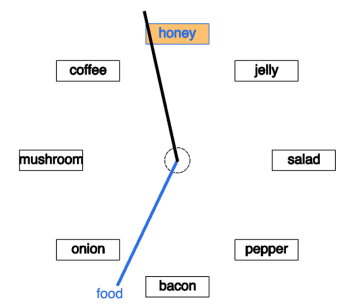

(b)
Figure 2. Representation of menus and commands in the context of selections by directional gestures in novice mode. The user's gesture is represented by a black line, and a blue line represents a valid menu selection (b).

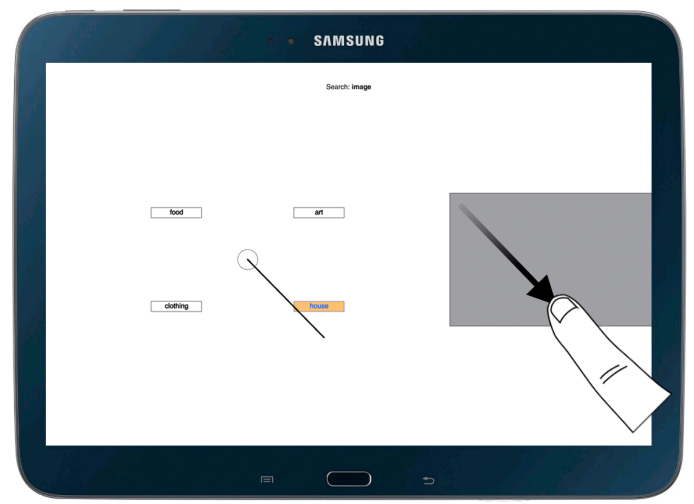

Figure 3. Example of a gesture performed by the user on the dedicated interaction zone to avoid the occlusion of commands.

Once the users know the positions of the commands, they no longer need to see their labels and can use the expert mode. Selections are performed in the same way as in novice mode. To avoid pointing errors, the closest command from the pointed location is selected.

Direction. We use an interaction technique strongly inspired by the Simple-mark menus [37] to test directional gestures. This variant of hierarchical Marking menus [20] has the advantage of minimizing selection errors [37]. It relies on a two-step selection mechanism. The first directional gesture selects the menu and the second gesture selects the desired command.

It is worth noticing that the techniques used for positional and directional gestures thus both rely on a two-step selection mechanism, which makes them very similar, except that they do not use the same gestures. This is another reason why we used Simple-mark menus rather than compound hierarchical Marking menus.

The novice mode is triggered by pressing for one second without moving (Figure 2-a). The users can then make a gesture (represented by a black line in Figure 2-b) in the direction of the menu they want to select. Once a menu is selected, a blue line shows the gesture that was performed (Figure 2-b). Performing a second directional gesture selects the desired command in this menu (Figure 2-b). As expected, the labels of the commands are not displayed in expert mode (but black and blue lines as still displayed). A gesture smaller than the limit indicated by the central circle on Figure 2 cancels a menu selection.
In order to not disadvantage this technique compared to the technique using positional gestures, the user performs directional gestures in a dedicated zone located on the side of the device (Figure 3). Otherwise, the user could obscure the representation displayed on the screen while interacting as he is continuously touching the screen with his finger. The users can start a gesture from any location inside this area.

\section{USER STUDY}

In this section, we present a study comparing the memorization of sets of commands using the two interaction techniques described in the previous section (Positions and Directions, factor Tесн). Our main working hypothesis is that since Positions are likely to benefit more from spatial memory, they should yield better results than Directions.

Participants. We analyzed the behavior of 16 participants (including 6 females) aged 23 to 40 years (mean $=28$ years) during the study.

Apparatus. A Samsung Galaxy Tablet Tab Pro 10.1 with a screen measuring $13.6 \times 21.8 \mathrm{~cm}$ was used for the experiment.

Evaluation sessions. The experiment was divided into three sessions spread over one to two weeks (mean $=9.33 \pm 2.79$ days). Each session was composed of learning phases and recall phases (Figure 4). The first session consisted of 3 pairs of learning and recall phases to assess mid-term memory (L1R1, L2-R2, L3-R3). The second session, one day later, started with a recall phase (R4) to assess users' information retention, followed by two phases (L4-R5) to reinforce their knowledge. Finally, a third session consisting of a single recall phase (R6) was performed one to two weeks later to evaluate long-term memory (only one subject could not do this last session).

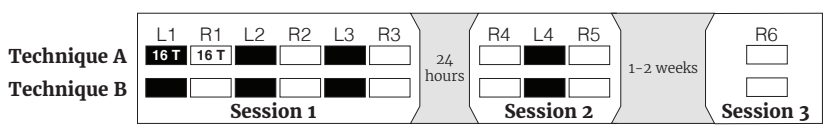

Figure 4. Experimental design of the study: the three sessions are held over one to two weeks with learning (L) and recall (R) phases consisting of 16 trials each.

During the learning phases, participants had to memorize how to select commands. These phases always started in expert mode (i.e., command labels not displayed), so that users had to trigger the novice mode explicitly. Hence, participants that already remembered the proper gestures could perform this stage in expert mode. This feature allowed us to evaluate how often users had to enter the novice mode.

As with Marking menus, we used a delay for entering the novice mode, but this delay was a bit longer (one second) to be sure that participants would not trigger the novice mode by mistake. During the recall phases, only the expert mode was available, forcing participants to recall previous selections.

The participants had to press a button in the center of the screen to start a trial. The name of the command to select was then displayed at the top of the screen (Figure 3). Subjects were asked to memorize as many commands as possible. During the learning phase trials, sound feedback was played after a command was selected to indicate its validity. In the case of a 

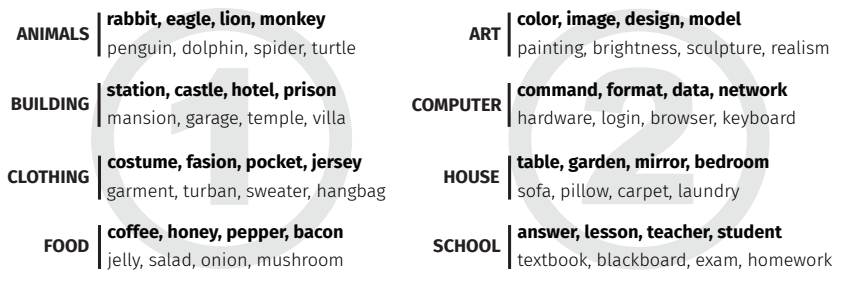

Figure 5. The two sets of order categories used during the study. The bold commands had to be memorized by the subjects.

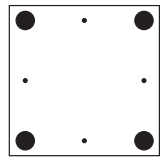

Extreme

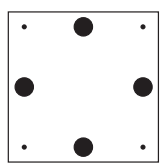

Figure 6. Positions of commands

mands to be memorized for each category. Each line was associated with one or the other technique for a given participant.

wrong selection, the novice mode was triggered automatically, and the subject was forced to select the correct command. During the recall phases, the participants could select only one command, and no sound was played when selecting it. At the end of each recall phase, the recall rates that were previously obtained were revealed to the participants to inform them of their performance.

Experimental design. We used a within-subject design to compare the two interaction techniques. All phases of a session were performed one after the other using an interaction technique, then the next (Figure 4). Before starting the phases of the first session with a technique, a familiarization phase composed of 8 trials using a different vocabulary was carried out by the participants so that they were sufficiently accustomed to the technique. To avoid an order effect between techniques, the order of the techniques was counterbalanced from one subject to another.

Categories. Two sets of four command categories were created for the study (Figure 5). Each set was balanced between the techniques to avoid a bias on the performance of the techniques. These categories were composed of 8 orders each. To create the categories, we chose frequent words from the English language ${ }^{2}$, not sharing the same initials. For each phase, half of the most frequent words in each category had to be memorized. The position of the categories for each technique was balanced between the subjects.

Command positions. As participants only had to memorize half of each category, we used specific patterns to position the commands to memorize. As shown in Figure 6, the patterns used are two by two complementary. Using these patterns made it possible to vary the proximity of the targeted commands to prevent an uncontrolled effect of their positions on

\footnotetext{
${ }^{2}$ list of frequent English words https://en.wiktionary .org/wiki/ Wiktionary: Frequency_lists\#English
}

recall rates and to assess the benefit of this proximity. The rest of the positions were used to display commands representing distractors. Each line in Figure 6 represents a group of patterns associated with one of the two techniques for a given subject. The positions of the patterns were counter-balanced across subjects.

Learning time. Since learning time has an essential effect on memorization, we decided to limit it for all participants in order to avoid too large differences. To do this, once the novice mode was triggered, the commands gradually vanished for 10 seconds (they were then invisible). The subjects thus had to memorize the commands during this period of time.

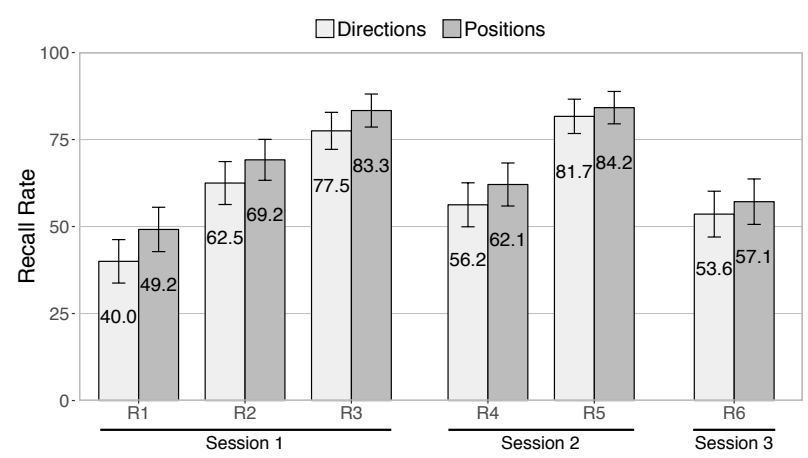

Figure 7. Recall rate by TECH over the three sessions.

\section{Results}

By comparing the results obtained by all participants, it turned out that the results of P11 were abnormally different (more than 1.5 times the interquartile range) on phases $\mathrm{R} 1, \mathrm{R} 4$, and $\mathrm{R} 5$, as well as very different from the rest of the participants on phase R6. Therefore, we do not consider the results obtained by this subject in the analyses presented below. To compare two sets of data we use in the following Wilcoxon's signed rank tests [34]. The confidence intervals on the graphs represent $95 \%$.

Recall Rate. The recall rates obtained using TECH are shown in Figure 7. Performance is quite high at the end of the first session (R3) with rates of $77.5 \%$ for Directions and $83.3 \%$ for Positions. They decrease significantly after 24 hours (R4: $56.2 \%$ vs. $62.1 \%$ ) but then remain fairly stable (R6: $53.6 \%$ vs. $57.1 \%$ ) after a period of one to two weeks. These results seem to indicate an advantage of Positions, with for example an improvement of $10.5 \%$ and $6.5 \%$ in retention phases R4 and R6, but differences are not significant ( $p$ 's $>0.1$ ).

Concerning the patterns used to position the commands (Figure 8), in the case of Positions, the extreme positions (i.e., corners and edges) allow much better performance $(27.1 \%$ improvement, $p<0.001$ ), which suggests the use of distant and regular spatial reference points.

Novice mode activation. The choice was left to the participants whether to use the novice mode during the learning phases. A comparison of the activation rate of the novice mode for each TECH shows that the participants used it less often with Positions than with Directions (72.5\% activation against $83.8 \%$ for A2, $53.3 \%$ vs. $64.2 \%$ for A3, and $55.8 \%$ vs. $66.7 \%$ for 


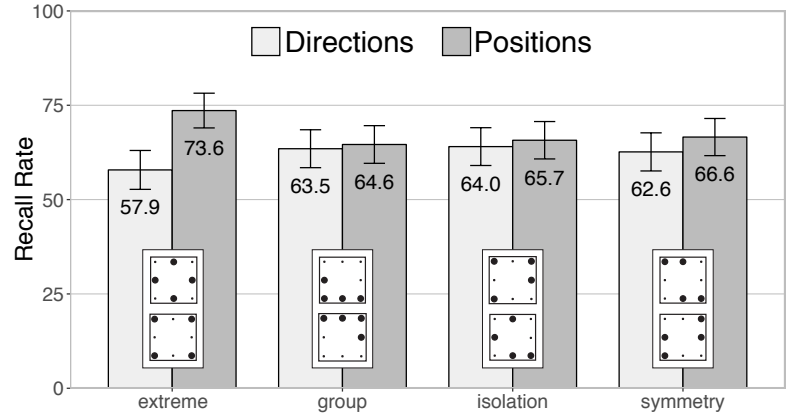

Figure 8. Average recall rate obtained over all phases for each command position pattern relative to the $\mathrm{TECH}$ used.

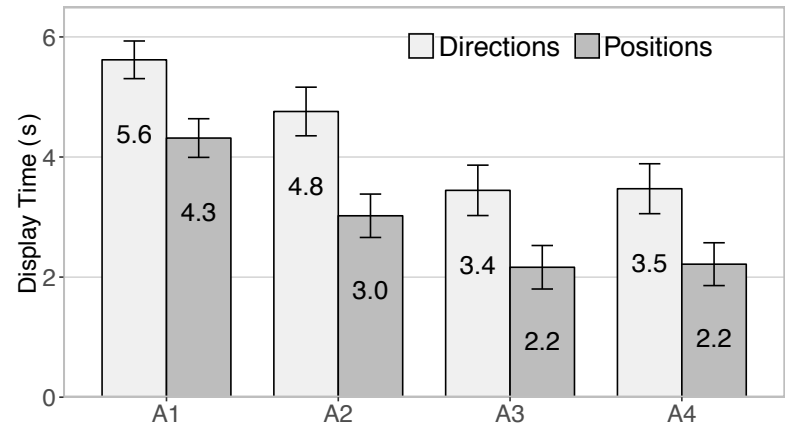

Figure 9. Average command display times during learning phases for each TECH.

A $4 ; p$ 's $<0.01)$. This suggests that using Positions requires fewer repetitions to encode information in memory than using Directions.

Learning time. Time is an important factor in learning and retaining information. The average display time (how long the commands were displayed in the learning phases) for each TECH is shorter with Positions, with a difference varying between $1.70 \mathrm{~s}$ and $1.25 \mathrm{~s}$ depending on the phases ( $p$ 's $<0.001$, Figure 9). This result, which points in the same direction than relying less on the novice mode when using positions, seems to indicate that commands are learned more quickly by encoding spatial positions in memory.

Questionnaire. At the end of the first session, subjects had to fill out a form based on the NASA TLX model [19]. The Likert's scales in this questionnaire were set from 1 (very

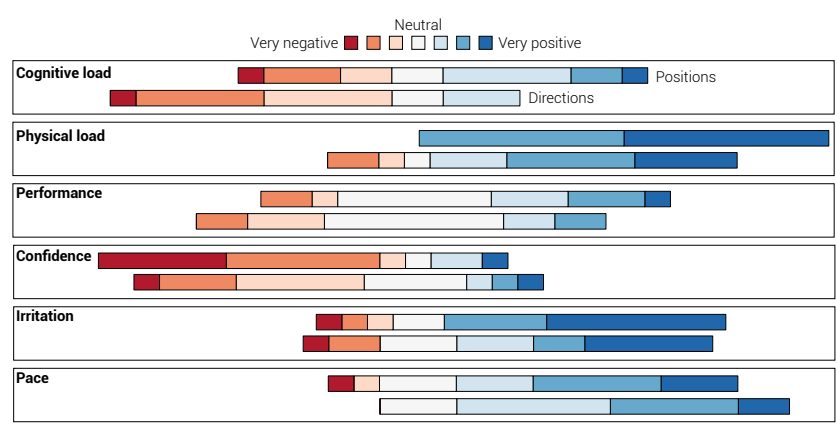

Figure 10. Responses to the questionnaire submitted to the subjects. negative) to 7 (very positive). Their results are shown in Figure 10. In addition to these scales, subjects were asked if they preferred one of the two ТЕсн and what strategies they developed during the experiment to memorize the commands. Preferences were mixed but in favor of Positions, with 9 subjects preferring using them ("Seemed more structured to me, easier to remember" P16) and 5 for Directions ("It was easier to remember for me. I think doing the gesture helped." P3), one subject preferring neither technique. The analysis of the results obtained using Likert's scales suggests an advantage of Positions in cognitive $(p=0.014)$ and physical $(p=0.014)$ loads, as well as a tendency for their confidence in their memorization abilities $(p=0.085)$. This better appreciation of Positions in terms of effort suggests that this modality is easier to handle than Directions. This result partly explains why Directions led to higher completion times.

Strategies. We categorized the strategies described by participants during the questionnaire and found similarities. First, two-thirds of the subjects (66.6\%) decided to group several commands according to their positions, or their meaning (e.g. "the teacher is asking a student for an answer" P15). This first observation indicates the importance of command positions in an interface, including their relative positions [8]. The positions of the commands are all more important as $26.6 \%$ of the subjects reported memorizing the patterns formed by these positions, without having been informed of their existence. Finally, more than half of the subjects $(60 \%)$ reported memorizing commands using their meaning (e.g., "the eagle is up because it flies" P1 or "bacon goes down into the belly, so down" P8).

\section{DISCUSSION}

Recall Rate. First of all, the performance in terms of memorization is quite high: $77.5 \%$ for Directions and $83.3 \%$ for Positions at the end of the first session and more than half of the commands were retained during the retention phases $\mathrm{R} 4$ (24 hours after) and R6 (1 to 2 weeks after) with rates of 56.2\% and $53.6 \%$ for Directions and $62.1 \%$ and $57.1 \%$ for Positions. It is interesting to note that in both cases performance remains fairly stable between the two retention phases. Presumably, due to individual variability, we did not find any significant differences in each phase ( $p$ 's $>0.1)$.

The strategies used by the participants to memorize the commands were often similar in both cases $(33.3 \%$ of subjects explained it), which probably contributes to the similarity in performance. The advantages attributed to spatial memory also seem to have been used for directional gestures, judging by these strategies (grouping commands, memorizing position patterns). This result, however, may be strongly linked to the type of the learning task (i.e., paired-learning), that allowed participants to focus on the way they memorize commands. The movements performed in both conditions had similar lengths (directions - $2.68 \pm 1.04 \mathrm{~cm}$; positions $-2.79 \pm 1.20 \mathrm{~cm}$ ). This suggests that motor learning is unlikely to have a much stronger effect in one case than in the other.

Spatial landmarks. Using several command patterns (Figure 6), we observed that the commands placed in the corners 
and on the sides obtained better recall rates (27.1\% improvement, $p<0.001$ ) for the positions (see the "extreme" pattern). We verified that this result was not due to learning one category better than another. Therefore, it indicates that a regular pattern using separate spatial cues can help the user in his task of memorization, as other studies seem to indicate, such as $[8,24,32]$. Thus, the lower recall rates obtained with the directions can perhaps be explained by the lack of explicit spatial landmarks inherent in this type of technique.

Advantages of Positions. The novice mode was used more often in all learning phases with directions and participants spent more time learning them $(\approx 1.5 \mathrm{~s}$ of difference), which should have produced a difference in memory performance. However, they did not perform better with this modality, thus suggesting that spatial positions are encoded faster in memory. Different execution times for both techniques could also explain this result. However, differentiating execution and reaction times precisely is a complex task, that is hardly possible in this sort of experiment because they are mixed. For instance, when performing positional gestures, the user may still be trying to remember the exact gesture while moving his hand. Moreover, as both techniques use a two-step selection mechanism, users may perform the first gesture, then do a short pause, then perform the second gesture. Finally, the results of the questionnaire suggest that participants needed less effort to memorize positions. All of these observations suggest a benefit to learning positions relatively to directions.

\section{Research Leads and Suggestions}

Command grouping. We observed that $66.6 \%$ of the subjects formed groups of commands to facilitate memorization. This type of strategy has also been observed in other studies [7, 13, 24]. It is interesting to note that the majority of participants used this method without being instructed to do so. To allow all participants to benefit from such methods, graphical interfaces could provide techniques for better highlighting groups of related commands, possibly using geometrical or spatial hints rather than using long lists or menus.

Semantics. Most of the commands used during our study belonged to categories referring to concrete concepts (e.g., animals, plants). These concepts promoted the use of semantic memory, as $60 \%$ of participants reported. A few participants said they had more difficulty memorizing the items of more abstract categories such as art or computer and (however this was not supported by our results). Since application commands usually refer to abstract concepts, associating images [15] or peg words [23] to commands may help users learning such commands. However, an issue with images (e.g., icons), is that their concreteness may be difficult to judge, and this approach has proved ineffective in some previous studies [13, 32].

Placement of commands. To recall commands, participants relied on their relative positions inside a category, sometimes also taking their meaning into account (e.g., "the eagle is $u p$ because it flies" P1, or "answer is up because it's good" P7). We observed for example that participants usually associated up and down to good/strong and bad/weak concepts. Thus, interactions between meaning and location may be worth considering, particularly when the commands evoke a concept or a direction, for instance, undo/redo commands might evoke past/future and left/right.

\section{CONCLUSION}

In this paper, we investigated the memorization of two types of gestural techniques, either based on directional gestures or positional gestures using spatially-stable arrangements of items. The goal of the study was to see whether memorization would show different characteristics for these two common types of gestures, as memorization may not involve the same memory components in both cases.

A study conducted in three sessions over a period of one to two weeks showed no significant differences in the memory performance between the two conditions. However, our analysis suggests several advantages of positional gestures for command learning that remains to be confirmed. These advantages could be amplified with a larger number of commands, i.e., with more menus or more commands inside menus (as suggested in [32]).

The analysis of the questionnaire completed by the participants revealed the spontaneous use of several strategies, such as command grouping or the use of semantics to facilitate memorization. These strategies seem to indicate an effective use of spatial memory for both types of gestures, with some advantages for positional gestures when spatial landmarks are used (which confirms [32]).

We also suggested some hints for improving the learning of commands by novice users (grouping and spatial landmarks, associating concrete concepts with abstract commands). The design of such user interfaces is an interesting problem that we would like to investigate in future work.

\section{ACKNOWLEDGMENTS}

This research was partially funded by Labex DigiCosme (ANR11-LABEX-0045-DIGICOSME), operated by the French Agence Nationale de la Recherche (ANR) as part of the program "Investissement d'Avenir" Idex Paris-Saclay (ANR-11-IDEX-0003-02).

\section{REFERENCES}

1. Caroline Appert and Shumin Zhai. 2009. Using strokes as command shortcuts: Cognitive benefits and toolkit support. In Proceedings of the SIGCHI Conference on Human Factors in Computing Systems (CHI '09). ACM, 2289-2298. 10.1145/1518701.1519052

2. A.D. Baddeley. 2013. Essentials of Human Memory (Classic Edition). Psychology Press, East Sussex, UK.

3. Gilles Bailly, Eric Lecolinet, and Laurence Nigay. 2008. Flower Menus: A New Type of Marking Menu with Large Menu Breadth, Within Groups and Efficient Expert Mode Memorization. In Proceedings of the Working Conference on Advanced Visual Interfaces (AVI '08). ACM, 15-22. 10.1145/1385569.1385575

4. Gilles Bailly, Eric Lecolinet, and Laurence Nigay. 2016. Visual menu techniques. ACM Comput. Surv. 49, 4, Article 60, 41 pages. 10.1145/3002171 
5. Olivier Bau and Wendy E. Mackay. 2008. OctoPocus: A Dynamic Guide for Learning Gesture-based Command Sets. In Proceedings of the 21st Annual ACM Symposium on User Interface Software and Technology (UIST '08). ACM, 37-46. 10.1145/1449715.1449724

6. Thomas Baudel and Michel Beaudouin-Lafon. 1993. Charade: Remote control of objects using free-hand gestures. Commun. ACM 36, 7, 28-35. 10.1145/159544.159562

7. Joanna Bergstrom-Lehtovirta, Sebastian Boring, and Kasper Hornbæk. 2017. Placing and recalling virtual items on the skin. In Proceedings of the 2017 CHI Conference on Human Factors in Computing Systems (CHI'17). ACM, 1497-1507. 10.1145/3025453.3026030

8. Marvin M Chun and Yuhong Jiang. 1998. Contextual cueing: Implicit learning and memory of visual context guides spatial attention. Cognitive psychology 36, 1, 28-71.

9. Christopher Clarke, Alessio Bellino, Augusto Esteves, and Hans Gellersen. 2017. Remote control by body movement in synchrony with orbiting widgets: an evaluation of tracematch. Proceedings of the ACM on Interactive, Mobile, Wearable and Ubiquitous Technologies 1, 3, Article 45, 23 pages. 10.1145/3130910

10. Andy Cockburn, Per Ola Kristensson, Jason Alexander, and Shumin Zhai. 2007. Hard lessons: Effort-inducing interfaces benefit spatial learning. In Proceedings of the SIGCHI Conference on Human Factors in Computing Systems (CHI '07). ACM, 1571-1580.

10.1145/1240624.1240863

11. Christian F. Doeller and Neil Burgess. 2008. Distinct error-correcting and incidental learning of location relative to landmarks and boundaries. Proceedings of the National Academy of Sciences 105, 15, 5909-5914. 10.1073/pnas.0711433105

12. Bruno Fruchard, Eric Lecolinet, and Olivier Chapuis. 2017. MarkPad: Augmenting Touchpads for Command Selection. In Proceedings of the 2017 CHI Conference on Human Factors in Computing Systems (CHI'17). ACM, 5630-5642. 10.1145/3025453.3025486

13. Bruno Fruchard, Eric Lecolinet, and Olivier Chapuis. 2018a. Impact of semantic aids on command memorization for on-body interaction and directional gestures. In Proceedings of the 2018 International Conference on Advanced Visual Interfaces (AVI'18). ACM, Article 14, 9 pages. 10.1145/3206505.3206524

14. Bruno Fruchard, Eric Lecolinet, and Olivier Chapuis. 2018b. Mémorisation de commandes: Positions spatiales versus gestes directionnels. In Proceedings of the 30th international conference of the Association Francophone d'Interaction Homme-Machine (IHM '18). ACM, 8 pages. To appear.

15. Tovi Grossman, Pierre Dragicevic, and Ravin Balakrishnan. 2007. Strategies for accelerating on-line learning of hotkeys. In Proceedings of the SIGCHI Conference on Human Factors in Computing Systems (CHI '07). ACM, 1591-1600. 10.1145/1240624.1240865
16. Carl Gutwin and Andy Cockburn. 2006. Improving List Revisitation with ListMaps. In Proceedings of the Working Conference on Advanced Visual Interfaces (AVI '06). ACM, 396-403. 10.1145/1133265.1133347

17. Carl Gutwin, Andy Cockburn, and Benjamin Lafreniere. 2015. Testing the rehearsal hypothesis with two fasttap interfaces. In Proceedings of the 41st Graphics Interface Conference (GI '15). Canadian Information Processing Society, 223-231.

http: //dl. acm.org/citation. $\mathrm{cfm} ? \mathrm{id}=2788890.2788930$

18. Carl Gutwin, Andy Cockburn, Joey Scarr, Sylvain Malacria, and Scott C. Olson. 2014. Faster Command Selection on Tablets with FastTap. In Proceedings of the $32 N d$ Annual ACM Conference on Human Factors in Computing Systems (CHI'14). ACM, 2617-2626. $10.1145 / 2556288.2557136$

19. Sandra G. Hart and Lowell E. Staveland. 1988. Development of NASA-TLX (Task Load Index): Results of empirical and theoretical research. In Human Mental Workload, Peter A. Hancock and Najmedin Meshkati (Eds.). Advances in Psychology, Vol. 52. North-Holland, 139 - 183. 10.1016/S0166-4115(08)62386-9

20. Gordon Kurtenbach and William Buxton. 1993. The limits of expert performance using hierarchic marking menus. In Proceedings of the INTERCHI '93 Conference on Human Factors in Computing Systems (INTERCHI '93). IOS Press, 482-487.

http://dl . acm.org/citation. cfm?id=164632 . 164977

21. Jean M Mandler, Dale Seegmiller, and Jeanne Day. 1977. On the coding of spatial information. Memory \& Cognition 5, 1, 10-16.

22. Miguel A. Nacenta, Yemliha Kamber, Yizhou Qiang, and Per Ola Kristensson. 2013. Memorability of Pre-designed and User-defined Gesture Sets. In Proceedings of the SIGCHI Conference on Human Factors in Computing Systems (CHI'13). ACM, 1099-1108.

$10.1145 / 2470654.2466142$

23. Allan Paivio. 1965. Abstractness, imagery, and meaningfulness in paired-associate learning. Journal of Verbal Learning and Verbal Behavior 4, 1, 32 - 38. 10.1016/S0022-5371(65)80064-0

24. Simon T. Perrault, Eric Lecolinet, Yoann Pascal Bourse, Shengdong Zhao, and Yves Guiard. 2015. Physical loci: Leveraging spatial, object and semantic memory for command selection. In Proceedings of the 33rd Annual ACM Conference on Human Factors in Computing Systems (CHI'15). ACM, 299-308. 10.1145/2702123.2702126

25. George Robertson, Mary Czerwinski, Kevin Larson, Daniel C. Robbins, David Thiel, and Maarten van Dantzich. 1998. Data mountain: Using spatial memory for document management. In Proceedings of the 11th Annual ACM Symposium on User Interface Software and Technology (UIST'98). ACM, 153-162.

10.1145/288392.288596 
26. Anne Roudaut, Gilles Bailly, Eric Lecolinet, and Laurence Nigay. 2009. Leaf Menus: Linear Menus with Stroke Shortcuts for Small Handheld Devices. In Proceedings of the 12th IFIP TC 13 International Conference on Human-Computer Interaction: Part I (INTERACT '09). Springer-Verlag, 616-619.

10.1007/978-3-642-03655-2_69

27. Vit Rusnak, Caroline Appert, Olivier Chapuis, and Emmanuel Pietriga. 2018. Designing coherent gesture sets for multi-scale navigation on tabletops. In Proceedings of the 36th international conference on Human factors in computing systems (CHI'18). ACM, 142:1-142:12. 10.1145/3173574.3173716

28. Joey Scarr, Andy Cockburn, Carl Gutwin, and Andrea Bunt. 2012. Improving command selection with CommandMaps. In Proceedings of the SIGCHI Conference on Human Factors in Computing Systems (CHI'12). ACM, 257-266. 10.1145/2207676.2207713

29. Joey Scarr, Andy Cockburn, Carl Gutwin, and Sylvain Malacria. 2013. Testing the robustness and performance of spatially consistent interfaces. In Proceedings of the SIGCHI Conference on Human Factors in Computing Systems (CHI'13). ACM, 3139-3148.

$10.1145 / 2470654.2466430$

30. Richard A. Schmidt, Timothy Donald Lee, and others. 2005. Motor control and learning: A behavioral emphasis. Vol. 4. Human kinetics, Champaign, IL, USA.

31. Endel Tulving and others. 1972. Episodic and semantic memory. Organization of memory 1, 381-403.

32. Md. Sami Uddin, Carl Gutwin, and Andy Cockburn. 2017. The Effects of Artificial Landmarks on Learning and Performance in Spatial-Memory Interfaces. In Proceedings of the 2017 CHI Conference on Human Factors in Computing Systems (CHI'17). ACM, 3843-3855. 10.1145/3025453.3025497

33. Md. Sami Uddin, Carl Gutwin, and Benjamin Lafreniere. 2016. Handmark menus: Rapid command selection and large command sets on multi-touch displays. In Proceedings of the 2016 CHI Conference on Human Factors in Computing Systems (CHI'16). ACM, 5836-5848. 10.1145/2858036.2858211

34. Frank Wilcoxon. 1945. Individual comparisons by ranking methods. Biometrics bulletin 1, 6, 80-83.

35. Frances Yates. 1992. The Art of Memory. Random House UK, London.

36. Shengdong Zhao, Maneesh Agrawala, and Ken Hinckley. 2006. Zone and polygon menus: Using relative position to increase the breadth of multi-stroke marking menus. In Proceedings of the SIGCHI Conference on Human Factors in Computing Systems (CHI '06). ACM, 1077-1086. 10.1145/1124772.1124933

37. Shengdong Zhao and Ravin Balakrishnan. 2004. Simple vs. Compound Mark Hierarchical Marking Menus. In Proceedings of the 17th Annual ACM Symposium on User Interface Software and Technology (UIST'04). ACM, 33-42. 10.1145/1029632.1029639

38. Jingjie Zheng, Xiaojun Bi, Kun Li, Yang Li, and Shumin Zhai. 2018. M3 gesture menu: Design and experimental analyses of marking menus for touchscreen mobile interaction. In Proceedings of the 2018 CHI Conference on Human Factors in Computing Systems (CHI'18). ACM, Article 249, 14 pages. 10.1145/3173574.3173823 